\author{
16 See Ref. 19, p. 742. \\ 17 National Bureau of Standards, Tables Relating to Mathieu \\ Functions (Columbia U.P., New York, 1951). \\ ${ }^{48}$ The conversion procedure is described in Ref. 47. \\ 40 This result is proved in Ref. 22, p. 52. \\ so Since we are defining the ${ }_{3} F_{2}(1)$ function $(4.7)$ by analytic \\ continuation, the condition $\operatorname{Re}(s)>0$ in Eq. (4.4) may be re- \\ laxed. \\ ${ }^{51}$ The contiguous function relations for generalized hypergeo- \\ metric functions have been discussed in detail by $E$. D. Rainville \\ in Ref. 35, p. 80. The required result Eq. (4.24) is a particular case \\ of Rainville's relation (15), p. 82. \\ ${ }^{82}$ This observation will be discussed in more detail in Paper II. \\ ${ }^{58}$ See Ref. 38, p. 34. \\ s4 R. J. Duffin, Duke Math. J. 20, 233 (1953). \\ ${ }_{55}$ H. Davies, Quart. J. Math. (Oxford) 6, 232 (1955). \\ ${ }^{56}$ M. Yussouff and J. Mahanty, Proc. Phys. Soc. 87, 689 \\ (1966). \\ ${ }^{52}$ The asymptotic expansion (5.10) was derived using a general \\ technique developed by Montroll and Weiss (see Ref. 12, Appendix \\ A). However, their final expression for $P(l, 1)$ is incorrect due to the \\ omission of a "weighting factor" which counts the "effective" \\ number of singularities in the region of integration. (For the simple
}

cubic, body-centered cubic, and face-centered cubic lattices this weighting factor is 1,4 , and 2 , respectively.)

${ }^{58}$ W. N. Bailey, Proc. Math. Soc. (London) 40, 37 (1935).

${ }^{50}$ See Ref. 23, p. 111.

${ }^{60}$ For details see Ref. 38 , p. 86.

${ }^{61}$ The analytic continuation (6.27) could also be established directly using a Barnes' contour integral representation for (6.22). ${ }^{62}$ A detailed discussion of this technique is given in Sec. 3 .

${ }^{63}$ The first application of the method of Darboux in lattice statistics appears to have been made by B. W. Ninham, J. Math. Phys. 4, 679 (1963).

${ }_{64}^{6}$ C. Domb, Proc. Cambridge Phil. Soc. 50, 586 (1954).

${ }^{85} \mathrm{~A}$. Dvoretzky and E. Erdös, in Proceedings of the Second Berkeley Symposium on Mathematical Statistics and Probability

(University of California Press, Berkeley, Calif., 1951), p. 353. ${ }_{6 B}$ G. H. Vineyard, J. Math. Phys. 4, 1191 (1963).

${ }^{67}$ C. Domb, J. Phys. C 3, L85 (1970).

${ }^{68}$ M. E. Fisher, Rept. Progr. Phys. 30, 615 (1967).

$0^{\circ}$ S. Katsura and T. Horiguchi, J. Math. Phys. 12, 230 (1971).

${ }^{70}$ G. Iwata, Natl. Sci. Rept., Ocanomizu Univ., Tokyo, 20, 13 (1969).

${ }^{11}$ T. Horiguchi and T. Morita, Phys. Letters 32A, 191 (1970).

72 G. S. Joyce, J. Phys. C 4, L53 (1971).

\title{
Quadratic Fermion Interaction Hamiltonian
}

\author{
Basilis GIDAS \\ Physics Department, University of Michigan, Ann Arbor, Michigan 48104
}

(Received 23 September 1970)

\begin{abstract}
The interaction Hamiltonian $\lambda \int: \bar{\psi}^{(0)}(\mathbf{x}) \psi^{(0)}(\mathbf{x}): g(\mathbf{x}) d^{s} \mathbf{x}, g(\mathbf{x}) \in \mathrm{S}\left(\Omega^{s}\right)$ is studied. An ultraviolet cutoff is introduced. We remove this cutoff, and take the limit $g \rightarrow 1$ in $S\left(\Re^{8}\right)$, by working with the Heisenberg fields. The limiting fields are well defined on the Fock space associated with the bare mass $m_{0}$. In the limit we get a new representation of the canonical anticommutation relations which is given by a (generalized) Bogoliubov transformation. The new representation is not always unitarily equivalent to the bare mass Fock representations.
\end{abstract}

\section{INTRODUCTION}

The existence of many inequivalent representations of the canonical anticommutation relations (CAR) was pointed out by Friedrichs ${ }^{1}$ and van $\mathrm{Hove}^{2}$; it was treated rigorously by Gårding and Wightman, ${ }^{3}$ Wightman and Schweber, ${ }^{4}$ and Golodes. ${ }^{5}$ It is well known by now that there is an uncountable number of inequivalent representations of the CAR which are both the hope and the harm of the Hamiltonian approach to quantum field theory. The problem is to find the right representation which makes bona fide a given Hamiltonian. The point is that when one works in the Fock space, translations are not unitarily implementable because of Haag's theorem ${ }^{6}$ and/or ultraviolet divergences. ${ }^{7}$ The usual approach to find the "correct" representations is to butcher the Hamiltonian by introducing enough cutoffs to develop a well-defined theory in the Fock space, and then try to recover the correct theory by some limiting procedure. This approach has been suggested by Wightman $^{7}$ and forms the nucleus of the work of Glimm and Jaffe. $^{8}$

In this note we exemplify Wightman's suggestion in the quadratic fermion interaction Hamiltonian. The method is the same one used by Guenin and Velo. ${ }^{\theta}$

For space-time dimensions $s+1$, this model leads to a new representation of the CAR which is given by a (generalized for $s+1 \geq 4$ ) Bogoliubov transformation. For $s+1 \geq 4$ in finite or infinite volume, and for $s=2$ in infinite volume, the new representation of the CAR is equivalent to the bare mass Fock representation. In all other cases the two representations are equivalent.

In Ref. 10 the same model has been studied by Glimm's method ${ }^{11}$ in the form used by Hepp ${ }^{12}$ and Fabrey. ${ }^{13}$ 
The model leads to linear field equations whose solution is trivial. However, working with the Hamiltonian, the model is far from trivial.

\section{FORMAL AND CUTOFF HAMILTONIAN}

We consider a Dirac field of bare mass $m_{0}$ in $(s+1)$-dimensional space-time whose free Hamiltonian $H_{0}$ is

$$
\begin{aligned}
H_{0} & =\int d^{s} s: \bar{\psi}^{(0)}(\mathbf{x})\left(-i \gamma^{*}+m_{0}\right) \psi^{(0)}(\mathbf{x}): \\
& =\sum_{r} \int d \mathbf{p} \omega(\mathbf{p})\left[a^{*}(\mathbf{p}, r) a(\mathbf{p}, r)+b^{*}(\mathbf{p}, r) b(\mathbf{p}, r)\right],
\end{aligned}
$$
where

$$
\begin{gathered}
\psi^{(0)}(\mathbf{x})=\frac{1}{(2 \pi)^{s / 2}} \int \frac{d \mathbf{p}}{\sqrt{ } \omega_{\mathbf{p}}}\left(\sum_{r} a(\mathbf{p}, r) u\left(\mathbf{p}, r ; m_{0}\right) e^{i \mathbf{p} \cdot \mathbf{x}}\right. \\
\left.+\sum_{r} b^{*}(\mathbf{p}, r) u\left(\mathbf{p}, r ; m_{0}\right) e^{-i \mathbf{p} \cdot \mathbf{x}}\right), \\
\bar{\psi}^{(0)}(\mathbf{x})=\frac{1}{(2 \pi)^{s / 2}} \int \frac{d \mathbf{p}}{\sqrt{ } \omega_{\mathbf{p}}}\left(\sum_{r} b(\mathbf{p}, r) \bar{u}\left(\mathbf{p}, r ; m_{0}\right) e^{i \mathbf{p} \cdot \mathbf{x}}\right. \\
\left.+\sum_{r} a^{*}(\mathbf{p}, r) \bar{u}\left(\mathbf{p}, r, m_{0}\right) e^{-i \mathbf{p} \cdot \mathbf{x}}\right), \\
\omega_{\mathbf{p}}=\omega(\mathbf{p})=\left(p^{2}+m_{0}^{2}\right)^{\frac{1}{2}}, \\
{\left[a(\mathbf{p}, r), a^{*}\left(\mathbf{p}^{\prime}, r^{\prime}\right)\right]_{+}=\delta\left(\mathbf{p}-\mathbf{p}^{\prime}\right) \delta_{r r^{\prime}},} \\
{\left[b(\mathbf{p}, r), b^{*}\left(\mathbf{p}^{\prime}, r^{\prime}\right)\right]_{+}=\delta(\mathbf{p}-\mathbf{p}) \delta_{r r^{\prime}} .}
\end{gathered}
$$

All other anticommutators are equal to zero. We summarize the properties of the Dirac spinors in an appendix. $H_{0}$ is a self-adjoint operator on the usual Fock space $\mathfrak{H}_{m_{0}}$, associated with a fermion of mass $m_{0} \cdot \psi^{(0)}(\mathbf{x})$ and $\bar{\psi}^{(0)}(\mathbf{x})$ are densely defined bilinear forms in $\mathscr{H}_{m_{0}} \times \mathscr{H}_{m_{0}}$, and bounded operators when smeared out with test functions in $L^{2}$.

We add now to $H_{0}$ a new term:

$$
\begin{aligned}
H_{I}= & \lambda \int: \bar{\psi}^{(0)}(\mathbf{x}) \psi^{(0)}(\mathbf{x}): d \mathbf{x} \\
= & \int d \mathbf{x} H_{I}(\mathbf{x}) \\
= & \lambda \sum_{r_{1}, r_{2}} \int d p\left[\frac{\bar{u}\left(\mathbf{p}, r_{1} ; m_{0}\right) u\left(-\mathbf{p}, r_{2} ; m_{0}\right)}{\left(\omega_{p} \omega_{p}\right)^{\frac{1}{2}}}\right. \\
& \times a^{*}\left(\mathbf{p}, r_{1}\right) b^{*}\left(-\mathbf{p}, r_{2}\right) \\
& +\frac{\bar{u}\left(\mathbf{p}, r_{1} ; m_{0}\right) u\left(+\mathbf{p}, r_{2} ; m_{0}\right)}{\omega_{\mathbf{p}}} a^{*}\left(\mathbf{p}, r_{1}\right) a\left(+\mathbf{p}, r_{2}\right) \\
& -\frac{\bar{u}\left(\mathbf{p}, r, m_{0}\right) u\left(\mathbf{p}, r_{2} ; m_{0}\right)}{\omega_{\mathbf{p}}} b^{*}\left(\mathbf{p}, r_{2}\right) b\left(+\mathbf{p}, r_{2}\right) \\
& \left.+\frac{\bar{u}\left(-\mathbf{p}, r_{1}\right) u\left(+\mathbf{p}, r_{2}\right)}{\sqrt{ } \omega_{\mathbf{p}}} b\left(-\mathbf{p}, r_{1}\right) a\left(\mathbf{p}, r_{2}\right)\right] \\
= & H_{I 2}+H_{I 1}+H_{I 0},
\end{aligned}
$$

which, formally, is the fermion mass renormalization. However, $H_{I}$, and therefore $H_{0}+H_{I}$, are not well defined on $\mathcal{H}_{m_{0}}$ for two reasons: first, because of a simple manifestation of Haag's theorem, namely, if we let $\Omega_{0}$ be the Fock vacuum, then

$$
\begin{aligned}
\left\|\left(H_{0}+H_{I}\right) \Omega_{0}\right\|^{2} & =\int_{x_{0}=y_{0}=0} d \mathbf{x} d \mathbf{y}\left[\Omega_{0}, H_{I}(\mathbf{x}) H_{I}(\mathbf{y}) \Omega_{0}\right] \\
& =\int d \mathbf{x} d \mathbf{y} F(\mathbf{x}-\mathbf{y})=\infty .
\end{aligned}
$$

In the second equality we have used translation invariance. Second, because of ultraviolet divergences, namely, if $g(x) \in \mathcal{D}$ is a form factor which takes care of the infinite volume divergence, then

$$
\begin{aligned}
& \left\|H_{I}(g) \Omega_{0}\right\|^{2} \\
& \quad \sim\left\|H_{I 2}(g) \Omega_{0}\right\|^{2} \\
& =\lambda^{2} \sum_{r_{1}, r_{2}} \int d p_{1} d p_{2}\left|\frac{\bar{u}\left(\mathbf{p}_{1}, r_{1} ; m_{0}\right) u\left(+\mathbf{p}_{2}, r_{2} ; m_{0}\right)}{\left(\omega_{\mathbf{p}_{1}} \omega_{\mathbf{p}_{2}}\right)^{\frac{1}{2}}}\right|^{2} \\
& \times\left|g\left(\mathbf{p}_{1}+\mathbf{p}_{2}\right)\right|^{2}
\end{aligned}
$$

diverges for $s \geq 1$ since $\left|\frac{u\left(\mathbf{p}_{1}, r_{1} ; m_{0}\right) u\left(\mathbf{p}_{2}, r_{2} ; m_{0}\right)}{\left(\omega_{p_{1}} \omega_{p_{2}}\right)}\right|$ is bounded. Thus we must introduce an ultraviolet cutoff $\kappa$. We do this by restricting the momentum integrations in $(-\kappa, \kappa)$. Then our cutoff Hamiltonian reads

$$
\begin{aligned}
H_{\kappa}(g)= & H_{0}+H_{I \kappa}(g) \\
= & H_{0}+\lambda \int d \mathbf{x}: \bar{\psi}_{\kappa}^{(0)}(\mathbf{x}) \psi_{\kappa}^{(0)}(\mathbf{x}): g(\mathbf{x}) \\
= & H_{0}+\lambda \int d \mathbf{p}_{1} d \mathbf{p}_{2} \hat{g}\left(\mathbf{p}_{1}+\mathbf{p}_{2}\right) \hat{x}_{\kappa}\left(\mathbf{p}_{1}, \mathbf{p}_{2}\right) \\
& \times\left(\frac{\bar{u}\left(\mathbf{p}_{1}, r_{1}\right) v\left(\mathbf{p}_{2}, r_{2}\right)}{\left(\omega_{\mathbf{p}_{1}} \omega_{\mathbf{p}_{2}}\right)^{\frac{1}{2}}} a^{*}\left(\mathbf{p}_{1}, r_{1}\right) b^{*}\left(\mathbf{p}_{2}, r_{2}\right)\right. \\
& +\frac{\bar{u}\left(-\mathbf{p}_{2}, r_{1}\right) u\left(\mathbf{p}_{1}, r_{2}\right)}{\left(\omega_{\mathbf{p}_{1}} \omega_{\mathbf{p}_{2}}\right)^{\frac{1}{2}}} a^{*}\left(\mathbf{p}_{1}, r_{2}\right) a\left(-\mathbf{p}_{2}, r_{2}\right) \\
& -\frac{\bar{v}\left(-\mathbf{p}_{2}, r_{1}\right) v\left(\mathbf{p}_{1}, r_{2}\right)}{\left(\omega_{\mathbf{p}_{1}} \omega_{\mathbf{p}_{2}}\right)^{\frac{1}{2}}} b^{*}\left(\mathbf{p}_{1}, r_{2}\right) b\left(-\mathbf{p}_{2}, r_{1}\right) \\
& +\frac{\bar{v}\left(-\mathbf{p}_{1} r_{1}\right) u\left(-\mathbf{p}_{2}, r_{2}\right)}{\left(\omega_{\mathbf{p}_{1}} \omega_{\mathbf{p}_{2}}\right)^{\frac{1}{2}}} \\
& \left.\times b\left(-\mathbf{p}_{1}, r_{1}\right) a\left(-\mathbf{p}_{2}, r_{2}\right)\right)
\end{aligned}
$$

where $\hat{g}$ is the Fourier transform

and

$$
\hat{g}(\mathbf{p})=\frac{1}{(2 \pi)^{s}} \int d \mathbf{x} e^{i \mathbf{p} \cdot \mathbf{x}} g(\mathbf{x}),
$$

$$
\begin{aligned}
\hat{x}_{\kappa}\left(\mathbf{p}_{1}, \mathbf{p}_{2}\right)=1 & \text { for }\left|\mathbf{p}_{1}\right|,\left|\mathbf{p}_{2}\right| \leq \kappa, \\
& =0 \text { otherwise. }
\end{aligned}
$$


Simple estimations show that $H_{I_{\kappa}}(g), \kappa<+\infty$, is a self-adjoint bounded operator for real $g(\mathbf{x})$. Thus, $H_{\kappa}(g)=H_{0}+H_{I_{\kappa}}(g), \quad \kappa<+\infty$, is a self-adjoint operator with domain $D\left[H_{\kappa}(g)\right]=D\left(H_{0}\right)$.

\section{HEISENBERG FIELDS}

Since $H_{\kappa}(g)$ is a self-adjoint operator, $e^{i t H_{\kappa}(g)}$ is a well-defined unitary operator. Thus we can define

$$
\psi_{\kappa g}(\mathbf{x}, i)=e^{i t H_{\kappa}(g)} \psi^{(0)}(\mathbf{x}) e^{-i t H_{\kappa}(g)} .
$$

Let us write

$$
\begin{aligned}
\psi_{\kappa}(\mathbf{x}, t)=\frac{1}{(2 \pi)^{s / 2}} \int & \frac{d p}{\sqrt{ } \omega_{p}}\left(\sum_{r} \tilde{a}_{g \kappa}(\mathbf{p}, r, t) u\left(\mathbf{p}, r, m_{0}\right) e^{i \mathbf{p} \cdot \mathbf{x}}\right. \\
& \left.+\sum \tilde{b}_{g \kappa}^{*}(\mathbf{p}, r, t) v\left(\mathbf{p}, r, m_{0}\right) e^{-i \mathbf{p} \cdot \mathbf{x}}\right)
\end{aligned}
$$

then

$$
\tilde{a}_{g \kappa}(\mathbf{p}, r, t)=e^{i t H_{\kappa}(g)} a(\mathbf{p}, r) e^{-i t H_{\kappa}(g)}
$$

satisfies the Heisenberg equations of motion, namely,

$$
i \frac{\partial \tilde{a}_{g \kappa}(\mathbf{p}, r, t)}{\partial t}=\left[\tilde{a}_{g \kappa}(p, r, t), H_{\kappa}(g)\right]
$$

with the initial conditions

$$
\tilde{a}_{g \kappa}(\mathbf{p}, r, 0)=a(\mathbf{p}, r), \quad \tilde{b}_{g \kappa}^{*}(\mathbf{p}, r, 0)=b(\mathbf{p}, r) .
$$

To solve these linear equations, we make the following ansatz:

$$
\begin{aligned}
\tilde{a}_{\kappa g}(\mathbf{p}, r, t)= & \sum_{r^{\prime}} \int K_{1}^{(\kappa g)}\left(t, \mathbf{p}, r, \mathbf{p}^{\prime}, r^{\prime}\right) a\left(\mathbf{p}^{\prime}, r^{\prime}\right) d \mathbf{p}^{\prime} \\
& +\sum_{r^{\prime}} \int K_{2}^{(\kappa, g)}\left(t, \mathbf{p}, r, \mathbf{p}^{\prime}, r^{\prime}\right) b^{*}\left(-\mathbf{p}^{\prime}, r^{\prime}\right) d \mathbf{p}^{\prime} .
\end{aligned}
$$

Substituting (2) into (1), we find that $K_{1}^{(x, g)}$ and $K_{2}^{(\kappa, g)}$ satisfy a system of integro-differential equations

$$
\begin{aligned}
& i \frac{\partial K_{1}^{(\kappa, g)}\left(t, \mathbf{p}, r, p^{\prime}, r^{\prime}\right)}{\partial t} \\
& =\omega\left(\mathbf{p}^{\prime}\right) K_{1}^{(\kappa, g)}\left(t, \mathbf{p}, r, p^{\prime}, r^{\prime}\right) \\
& +\lambda \int d \mathbf{p}^{\prime \prime} \hat{g}\left(\mathbf{p}^{\prime \prime}-\mathbf{p}^{\prime}\right) \chi_{\kappa}\left(\mathbf{p}^{\prime \prime},-\mathbf{p}^{\prime}\right) \\
& \times\left(\sum_{r^{\prime \prime}} \frac{\bar{u}\left(\mathbf{p}^{\prime \prime}, r^{\prime \prime} ; m_{0}\right) u\left(\mathbf{p}^{\prime}, r^{\prime} ; m_{0}\right)}{\left(\omega^{\prime} \omega^{\prime \prime}\right)^{\frac{1}{2}}}\right. \\
& \times K_{1}^{(\kappa, g)}\left(t, \mathbf{p}, r, \mathbf{p}^{\prime \prime}, r^{\prime \prime}\right) \\
& +\sum_{r^{\prime \prime}} \frac{v\left(-\mathbf{p}^{\prime \prime}, r^{\prime \prime}, m_{0}\right) u\left(\mathbf{p}^{\prime}, r^{\prime}, m_{0}\right)}{\left(\omega^{\prime} \omega^{\prime \prime}\right)^{\frac{1}{2}}} \\
& \left.\times K_{2}^{(\kappa, g)}\left(t, \mathbf{p}, r, \mathbf{p}^{\prime \prime}, r^{\prime \prime}\right)\right) \\
& i \frac{\partial K^{(\kappa, g)}\left(t, \mathbf{p}, r, \mathbf{p}^{\prime}, r^{\prime}\right)}{\partial t} \\
& =-\omega\left(\mathbf{p}^{\prime}\right) K_{2}^{(\kappa, g)}\left(t, \mathbf{p}, r, \mathbf{p}^{\prime}, r^{\prime}\right) \\
& +\lambda \int d \mathbf{p}^{\prime \prime} \hat{g}\left(\mathbf{p}^{\prime \prime}-\mathbf{p}^{\prime}\right) \chi_{\boldsymbol{\kappa}}\left(\mathbf{p}^{\prime \prime},-\mathbf{p}^{\prime}\right) \\
& \times\left(\sum_{r^{\prime \prime}} \frac{u\left(\mathbf{p}^{\prime \prime}, r^{\prime \prime}, m_{0}\right) v\left(-\mathbf{p}^{\prime}, r^{\prime}, m_{0}\right)}{\left(\omega^{\prime} \omega^{\prime \prime}\right)^{\frac{1}{2}}}\right. \\
& \times K_{1}^{(\kappa, g)}\left(t, \mathbf{p}, r, \mathbf{p}^{\prime \prime}, r^{\prime \prime}\right) \\
& +\sum_{r^{\prime \prime}} \frac{v\left(-\mathbf{p}^{\prime \prime}, r^{\prime \prime}, m_{0}\right) v\left(-\mathbf{p}^{\prime}, r^{\prime}, m_{0}\right)}{\left(\omega^{\prime} \omega^{\prime \prime}\right)^{\frac{1}{2}}} \\
& \left.\times K_{2}^{(\kappa, g)}\left(t, \mathbf{p}, r, \mathbf{p}^{\prime \prime}, r^{\prime \prime}\right)\right),
\end{aligned}
$$

with initial conditions

\section{Define}

$$
\begin{aligned}
& K_{1}^{(\kappa, g)}\left(0, \mathbf{p}, r, \mathbf{p}^{\prime}, r^{\prime}\right)=\delta\left(\mathbf{p}-\mathbf{p}^{\prime}\right) \delta_{r r^{\prime}}, \\
& K_{2}^{(\kappa, g)}\left(0, \mathbf{p}, r, \mathbf{p}^{\prime}, r^{\prime}\right)=0 .
\end{aligned}
$$

$$
K^{(\kappa, g)}\left(t, \mathbf{p}, \mathbf{p}^{\prime}, r^{\prime}\right)=\left(\begin{array}{c}
e^{i \omega_{p^{\prime}} t} K_{1}^{(\kappa, g)}\left(t, \mathbf{p}, r, \mathbf{p}^{\prime}, r^{\prime}\right) \\
e^{-i \omega_{p^{\prime}} t} K_{2}^{(\kappa, g)}\left(t, \mathbf{p}, r, \mathbf{p}^{\prime}, r^{\prime}\right)
\end{array}\right)
$$

and

$$
\begin{aligned}
& L^{(\kappa)}\left(t, \mathbf{p}^{\prime}, r^{\prime}, \mathbf{p}^{\prime \prime}, r^{\prime \prime}\right) \\
& =\frac{1}{\lambda} \chi_{k}\left(\mathbf{p}^{\prime \prime},-\mathbf{p}^{\prime}\right)\left(\begin{array}{cc}
e^{i\left(\omega^{\prime}-\omega^{\prime \prime}\right) t} \frac{\bar{u}\left(\mathbf{p}^{\prime \prime}, r^{\prime \prime} ; m_{0}\right) u\left(\mathbf{p}^{\prime}, r^{\prime} ; m_{0}\right)}{\omega^{\prime} \omega^{\prime \prime}}, & e^{i\left(\omega^{\prime}+\omega^{\prime \prime}\right) t} \frac{\bar{u}\left(-\mathbf{p}^{\prime \prime}, r^{\prime \prime}, m_{0}\right) u\left(\mathbf{p}^{\prime}, r^{\prime}, m_{0}\right)}{\omega^{\prime} \omega^{\prime \prime}} \\
e^{-i\left(\omega^{\prime}+\omega^{\prime \prime}\right) t} \frac{\bar{u}\left(\mathbf{p}^{\prime \prime}, r^{\prime \prime}, m_{0}\right) v\left(-\mathbf{p}^{\prime}, r^{\prime}, m_{0}\right)}{\omega^{\prime} \omega^{\prime \prime}}, & e^{-i\left(\omega^{\prime}-\omega^{\prime \prime}\right) t} \frac{\bar{v}\left(-\mathbf{p}^{\prime \prime}, r^{\prime \prime}, m_{0}\right) v\left(-\mathbf{p}^{\prime}, r^{\prime}\right)}{\omega^{\prime} \omega^{\prime \prime}}
\end{array}\right) .
\end{aligned}
$$

Then (3) can be written in the compact form

$$
\begin{aligned}
\frac{\partial}{\partial t} K^{(\kappa, a)} & \left(t, \mathbf{p}, r, \mathbf{p}^{\prime}, r^{\prime}\right) \\
= & \lambda \int d \mathbf{p}^{\prime \prime} \hat{g}\left(\mathbf{p}^{\prime \prime}-\mathbf{p}^{\prime}\right) \sum_{r^{\prime \prime}} L^{(\kappa)}\left(t, \mathbf{p}^{\prime}, r^{\prime}, \mathbf{p}^{\prime \prime}, r^{\prime \prime}\right) \\
& \times K^{(\kappa, g)}\left(t, \mathbf{p}, r, \mathbf{p}^{\prime \prime}, r^{\prime \prime}\right) .
\end{aligned}
$$

Theorem 1: (7) with initial conditions (4) has a unique solution, which, smeared out in $\mathbf{p}$ with test functions in $S\left(\mathcal{R}^{s}\right)$, belongs to $S\left(\mathcal{R}^{s}\right)$ in $\mathbf{p}^{\prime}$. Furthermore, this solution, when smeared out in p with test functions in $\delta\left(\mathcal{R}^{s}\right)$, converges in the $\delta$ topology as $\kappa \rightarrow+\infty$, and $g \rightarrow 1$ and the limit is the solution of (7) with $g=1$ and $\kappa=+\infty$. 
Proof: Let

Then

$$
f(p) \in \mathbf{S}\left(\Re^{s}\right)
$$

is a solution of

$$
K^{(\kappa, g)}\left(t, r, \mathbf{p}^{\prime}, r^{\prime}\right)=\int d \mathbf{p} f(\mathbf{p}) K^{(\kappa, g)}\left(t, \mathbf{p}, r, \mathbf{p}^{\prime}, r^{\prime}\right)
$$

$$
K^{(\kappa, g)}\left(t, r, \mathbf{p}^{\prime}, r^{\prime}\right)=K^{(\kappa, g)}\left(0, r, \mathbf{p}^{\prime}, r^{\prime}\right)+\int_{0}^{t} d t \int d \mathbf{p}^{\prime \prime} g\left(\mathbf{p}^{\prime \prime}-\mathbf{p}^{\prime}\right) \sum_{r^{\prime \prime}} L^{(\kappa)}\left(t, \mathbf{p}^{\prime}, r^{\prime}, \mathbf{p}^{\prime \prime}\right) K^{(\kappa, g)}\left(t, r, \mathbf{p}^{\prime \prime}, r^{\prime}\right)
$$

with initial condition

Iterating (8) we get the Neumann series

$$
K^{(\kappa, g)}\left(0, r, \mathbf{p}^{\prime}, r^{\prime}\right)=\left(\begin{array}{c}
\delta_{r r^{\prime}} f\left(\mathbf{p}^{\prime}\right) \\
0
\end{array}\right)
$$

$$
\begin{aligned}
& K^{\langle\kappa, g)}\left(t, r, \mathbf{p}^{\prime}, r^{\prime}\right) \\
&= K^{(\kappa, g)}\left(0, r, \mathbf{p}^{\prime}, r^{\prime}\right)+\sum_{n=1}^{+\infty} \lambda^{n} \int d \mathbf{p}_{1} \cdots d \mathbf{p}_{n} \int_{0}^{t} d t_{1} \int_{0}^{t_{1}} d t_{2} \cdots \int_{0}^{t_{n-1}} d t_{n} \hat{g}\left(\mathbf{p}_{1}-\mathbf{p}^{\prime}\right) \hat{g}\left(\mathbf{p}_{2}-\mathbf{p}_{1}\right) \cdots g_{n}\left(\mathbf{p}-\mathbf{p}_{n-1}\right) \\
& \times\left(\sum_{r_{1}, \cdots, r_{n}} L^{(\kappa)}\left(t_{1}, \mathbf{p}^{\prime}, r^{\prime}, \mathbf{p}_{1}, r_{1}\right) L^{(\kappa)}\left(t_{2}, \mathbf{p}_{1}, r_{1}, \mathbf{p}_{2}, r_{2}\right) \cdots L^{(\kappa)}\left(t_{n}, \mathbf{p}_{n-1}, r_{n-1} \mathbf{p}_{n}, r_{n}\right) K^{(\kappa, g)}\left(0, r, \mathbf{p}_{n}, r_{n}\right)\right)
\end{aligned}
$$

From (6) we have

$$
\left\|L^{(\kappa)}\left(t, \mathbf{p}, r, \mathbf{p}^{\prime}, r^{\prime}\right)\right\| \leq C_{1},
$$

where $C_{\mathbf{1}}$ is independent of $t, \mathbf{p}, r, \mathbf{p}^{\prime}, r^{\prime}$, and $\kappa$. Thus we get

$$
\begin{aligned}
& \left|K^{(\kappa, g)}\left(t, r, \mathbf{p}^{\prime}, r^{\prime}\right)\right| \\
& \quad \leq \sum_{n=0} \frac{C_{2}^{n} t^{n}}{n !}\left\|K^{(\kappa, g)}\left(0, r, \mathbf{p}^{\prime}, r^{\prime}\right)\right\|_{\infty},
\end{aligned}
$$

where the constant $C_{2}$ is independent of $t, \mathbf{p}, r, \mathbf{p}^{\prime}$, $r^{\prime}, k$, and $g$. Therefore the convergence of the Neumann series is uniform in $\mathbf{p}^{\prime}, \kappa$, and $g$. The same kind of estimates we can make for

$$
\left\|\left(\prod_{j=1}^{s} p_{j}^{\prime \alpha_{j}}\right)\left(\prod_{i=1}^{s} \frac{\partial^{p_{i}}}{\partial p_{i}^{\prime} p_{j}}\right) K^{(\kappa, g)}\left(t, r, p^{\prime}, r^{\prime}\right)\right\|,
$$

proving the convergence in $\delta\left(\mathfrak{R}^{s}\right)$ as $\kappa \rightarrow+\infty, g \rightarrow 1$.

\section{LIMITING SOLUTION AND THE ASSOCIATED BOGOLIUBOV TRANSFORMATION}

For $\kappa=+\infty$, and $g=1, g\left(\mathbf{p}^{\prime \prime}-\mathbf{p}^{\prime}\right)=\delta\left(\mathbf{p}^{\prime \prime}-\mathbf{p}^{\prime}\right)$,

(3) becomes

$$
\begin{aligned}
i \frac{\partial K_{1}\left(t, \mathbf{p}, r, \mathbf{p}^{\prime}, r^{\prime}\right)}{\partial t} & \\
= & -\omega\left(\mathbf{p}^{\prime}\right) K_{1}\left(t, \mathbf{p}, r, \mathbf{p}^{\prime}, r^{\prime}\right) \\
& +\frac{\lambda m_{0}}{\omega\left(p^{\prime}\right)} K_{1}\left(t, \mathbf{p}, r, \mathbf{p}^{\prime}, r^{\prime}\right) \\
& +\lambda \sum_{r^{\prime \prime}} \frac{\bar{v}\left(-\mathbf{p}^{\prime}, r^{\prime \prime}, m_{0}\right) u\left(\mathbf{p}, r^{\prime}, m_{0}\right)}{\omega^{\prime}} \\
& \times K_{2}\left(t, \mathbf{p}, r, \mathbf{p}^{\prime}, r^{\prime \prime}\right),
\end{aligned}
$$

$$
\begin{aligned}
i \frac{\partial K_{2}\left(t, \mathbf{p}, r, \mathbf{p}^{\prime}, r^{\prime}\right)}{\partial t} \\
=-\omega\left(\mathbf{p}^{\prime}\right) K_{2}\left(t, \mathbf{p}, r, \mathbf{p}^{\prime}, r^{\prime}\right) \\
\quad+\frac{\lambda m_{0}}{\omega^{\prime}} K_{2}\left(t, \mathbf{p}, r, \mathbf{p}^{\prime}, r^{\prime}\right) \\
\quad+\lambda \sum_{r^{\prime \prime}} \frac{\bar{u}\left(\mathbf{p}^{\prime}, r^{\prime \prime}, m_{0}\right) v\left(-\mathbf{p}^{\prime}, r^{\prime}, m_{0}\right)}{\omega^{\prime}} \\
\quad \times K_{1}\left(t, \mathbf{p}, r, \mathbf{p}^{\prime}, r^{\prime \prime}\right) .
\end{aligned}
$$

To solve these equations we make the ansatz

$$
\begin{aligned}
& K_{1}\left(t, \mathbf{p}, r, \mathbf{p}^{\prime}, r^{\prime}\right) \\
& \quad=A_{1}\left(\mathbf{p}, r, \mathbf{p}^{\prime}, r^{\prime}\right) e^{-i \Omega t}+A_{1}^{\prime}\left(\mathbf{p}, r, \mathbf{p}^{\prime}, r^{\prime}\right) e^{i \Omega t}, \\
& K_{\mathbf{2}}\left(t, \mathbf{p}, r, \mathbf{p}^{\prime}, r^{\prime}\right) \\
& \quad=A_{2}\left(\mathbf{p}, r, \mathbf{p}^{\prime}, r^{\prime}\right) e^{-i \mathbf{\Omega} t}+A_{2}^{\prime}\left(\mathbf{p}, r, \mathbf{p}^{\prime}, r^{\prime}\right) e^{i \mathbf{\Omega} t},
\end{aligned}
$$

where $\Omega(\mathbf{p})=\left(p^{2}+\left(m_{0}+\lambda\right)^{2}\right)^{\frac{1}{2}}$. Then

$$
\begin{aligned}
& K_{1}\left(t, \mathbf{p}, r, \mathbf{p}^{\prime}, r^{\prime}\right) \\
& =\left(\frac{\Omega \omega^{2}+\omega^{2}+\lambda m_{0}}{2 \Omega \omega} e^{-i \Omega t}+\frac{\Omega \omega^{2}-\omega^{2}-\lambda m_{0}}{2 \Omega \omega} e^{i \Omega t}\right) \\
& \times \delta\left(p-p^{\prime}\right) \delta_{r r^{\prime}},
\end{aligned}
$$

Theorem 2:

$$
\begin{aligned}
\tilde{a}_{\kappa g}(t, \mathbf{p}, r)= & \sum_{r^{\prime}} \int K_{1}^{(\kappa g)}\left(t, \mathbf{p}, r, \mathbf{p}^{\prime}, r^{\prime}\right) a\left(\mathbf{p}^{\prime}, r^{\prime}\right) d \mathbf{p}^{\prime} \\
& +\sum_{r^{\prime}} \int K_{2}^{(\kappa g)}\left(t, \mathbf{p}, r, \mathbf{p}^{\prime}, r^{\prime}\right) b^{*}\left(-\mathbf{p}^{\prime}, r^{\prime}\right) d \mathbf{p}^{\prime},
\end{aligned}
$$


when smeared out with test functions in $S\left(\Re^{s}\right)$, converges, as $k \rightarrow+\infty, g \rightarrow 1$, to

$$
\begin{aligned}
\tilde{a}(t, \mathbf{p}, r)= & \sum_{r^{\prime}} \int K_{1}\left(t, \mathbf{p}, r, \mathbf{p}^{\prime}, r^{\prime}\right) a\left(\mathbf{p}^{\prime}, r^{\prime}\right) d \mathbf{p}^{\prime} \\
& +\sum_{r^{\prime}} \int K_{2}\left(t, \mathbf{p}, r, \mathbf{p}^{\prime}, r^{\prime}\right) b^{*}\left(-\mathbf{p}^{\prime}, r^{\prime}\right) d \mathbf{p}^{\prime}
\end{aligned}
$$

in the norm topology of $\mathcal{L}\left(\mathscr{H}_{m_{0}}\right)$.

Proof: By Theorem 1, it suffices to prove that if $f_{n} \stackrel{S\left(R^{s}\right)}{\longrightarrow} f$, then $a^{\#}\left(f_{n}\right) \rightarrow a^{\#}(f)$ uniformly. Indeed $\left\|a^{\#}\left(f_{n}\right)-a^{\#}(f)\right\|=\left\|a^{\#}\left(f_{n}-f\right)\right\| \leq\left\|f_{n}-f\right\| \rightarrow 0$, where $\left\|f_{n}-f\right\|$ is some $S\left(\mathcal{R}^{s}\right)$ norm.

This theorem implies that $\psi_{\kappa g}(\mathbf{x}, t)$, when smeared out with functions in $L_{2}\left(\Re^{s}\right)$, converges uniformly to $\psi(\mathbf{x}, t)=\frac{1}{(2 \pi)^{s / 2}} \int \frac{d \mathbf{p}}{\sqrt{ } \omega_{\mathbf{p}}}$

$$
\times \sum_{r}\left(\tilde{a}(\mathbf{p}, r, t) u\left(\mathbf{p}, r, m_{0}\right) e^{i \mathbf{p} \cdot \mathbf{x}}\right.
$$

in $\mathcal{L}\left(\mathfrak{H}_{m_{0}}\right)$.

$$
\left.+\tilde{b}^{*}(\mathbf{p}, r, t) v\left(\mathbf{p}, r, m_{\mathbf{0}}\right) e^{-i \mathbf{p} \cdot \mathbf{x}}\right)
$$

After some simple manipulations we obtain

$$
\begin{aligned}
\psi(x, t)= & \frac{1}{(2 \pi)^{s / 2}} \int \frac{d \mathbf{p}}{(\Omega(\mathbf{p}))^{\frac{1}{2}}} \\
& \times\left(\sum_{r} \tilde{a}(\mathbf{p}, r) u(\mathbf{p}, r ; m) e^{-i \Omega t+i \mathbf{p} \cdot \mathbf{x}}\right. \\
& \left.\quad+\sum_{r} \tilde{b}^{*}(\mathbf{p}, r) v(\mathbf{p}, r, m) e^{i \Omega t-i \mathbf{p} \cdot \mathbf{x}}\right),
\end{aligned}
$$

where $m=m_{0}+\lambda$, and

$$
\begin{aligned}
\tilde{a}(\mathbf{p}, r) & =\sum_{r^{\prime}}\left(\frac{\bar{u}(\mathbf{p}, r ; m) \gamma^{0} u\left(\mathbf{p}, r^{\prime} ; m_{0}\right)}{(\omega \Omega)^{\frac{1}{2}}} a\left(\mathbf{p}, r^{\prime}\right)\right. \\
& \left.+\frac{\bar{u}(\mathbf{p}, r ; m) \gamma^{0} v\left(-\mathbf{p}, r^{\prime} ; m_{0}\right)}{(\omega \Omega)^{\frac{1}{2}}} b^{*}\left(-\mathbf{p}, r^{\prime}\right)\right), \\
\tilde{b}^{*}(-\mathbf{p}, r) & =\sum_{r^{\prime}}\left(\frac{\bar{u}(-\mathbf{p}, r ; m) \gamma^{0} u\left(\mathbf{p}, r^{\prime} ; m_{0}\right)}{(\Omega \omega)^{\frac{1}{2}}} a\left(p, r^{\prime}\right)\right. \\
& \left.+\frac{\bar{v}(-\mathbf{p}, r ; m) \gamma^{0} v\left(-\mathbf{p}, r^{\prime} ; m_{0}\right)}{(\Omega \omega)^{\frac{1}{2}}} b^{*}\left(-\mathbf{p}, r^{\prime}\right)\right) .
\end{aligned}
$$

In two- and three-dimensional space-time, the sum over $r^{\prime}$ reduces to a single term and the canonical transformation in (14) is the ordinary Bogoliubov transformation. For this transformation, it is known, Uhlenbrock, ${ }^{14}$ Ezawa, ${ }^{15}$ Klauder and McKenna, ${ }^{16}$ and Berezin, ${ }^{17}$ that the new representation is unitary equivalent to the original representation if and only if

$$
\int d^{s} \mathbf{p}\left|\frac{\bar{u}(\mathbf{p} ; m) \gamma^{0} v\left(-\mathbf{p} ; m_{0}\right)}{(\Omega \omega)^{\frac{1}{2}}}\right|^{2}<+\infty, \quad s=1,2 .
$$

After simple $\gamma$ gimmics, the integral can be written as

$$
\frac{1}{2} \int d^{s} \mathbf{p} \frac{\Omega(\mathbf{p}) \omega(\mathbf{p})-p^{2}-m m_{0}}{\Omega(\mathbf{p}) \omega(\mathbf{p})},
$$

which is convergent for $s=1$, but divergent for $s=2$.

In space of finite volume with periodic boundary conditions, criterion (15) reads

or

$$
\sum_{\mathbf{p}}\left|\frac{\bar{u}(\mathbf{p} ; m) \gamma^{0} v\left(-\mathbf{p}, m_{0}\right)}{(\Omega \omega)^{\frac{1}{2}}}\right|^{2}<+\infty
$$

$$
\frac{1}{2} \sum_{\mathbf{p}}\left(\frac{\Omega(\mathbf{p}) \omega(\mathbf{p})-\mathbf{p}^{2}-m m_{0}}{\Omega(\mathbf{p}) \omega(\mathbf{p})}\right)<+\infty,
$$

which is satisfied for $s=1,2$.

For $s \geq 3$, we get a generalized Bogoliubov transformation. This transformation has been studied in 10. This study shows that the transformation (14) is unitarily inequivalent to the Fock representation associated with a fermion of bare mass $m_{0}$.

\section{APPENDIX: CONVENTIONS AND SPINORS}

We use the metric

$$
g_{00}=1, \quad g_{i i}=-1, \quad g_{\mu \nu}=0 \text { for } \mu \neq \nu .
$$

The Dirac matrices satisfy

$$
\gamma_{\mu} \gamma_{\nu}+\gamma_{\nu} \gamma_{\mu}=2 g_{\mu \nu} \text {. }
$$

They form an irreducible Clifford algebra whenever $s$ is odd. We assume that the $\gamma^{\prime}$ 's are unitary and $\gamma_{0}^{*}=\gamma_{0}, \gamma_{j}^{*}=-\gamma_{j}$. We denote $u^{*}(\mathbf{p}) \gamma^{0}$ by $\bar{u}(\mathbf{p})$. The spinors satisfy

$$
\begin{array}{ll}
(\gamma \cdot p-m) u(\mathbf{p})=0, & \bar{u}(\mathbf{p})(\gamma \cdot p-m)=0 . \\
(\gamma \cdot p+m) v(\mathbf{p})=0, & \bar{v}(\mathbf{p})(\gamma \cdot p+m)=0 .
\end{array}
$$

They are normalized so that

$$
\begin{array}{ll}
u^{*}(\mathbf{p}, r) u(\mathbf{p}, s)=\omega_{p} \delta_{r s}, & \bar{u}(\mathbf{p}, r) u(\mathbf{p}, s)=m \delta_{r s}, \\
v^{*}(\mathbf{p}, r) v(\mathbf{p}, s)=\omega_{p} \delta_{r s}, & \bar{v}(\mathbf{p}, r) v(\mathbf{p}, s)=m \delta_{r s} .
\end{array}
$$

The orthogonality is expressed by

$$
\begin{gathered}
\sum_{r} u_{\alpha}(\mathbf{p}, r) \bar{u}_{\beta}(\mathbf{p}, r)=(\gamma \cdot p+m)_{\alpha \beta} / 2, \\
\sum_{r} v_{\alpha}(\mathbf{p}, r) \bar{v}_{\beta}(\mathbf{p}, r)=(\gamma \cdot p-m)_{\alpha \beta} / 2 .
\end{gathered}
$$

We need the following properties

$$
\begin{aligned}
\sum_{r, r^{\prime}}\left|u(\mathbf{p}, r) v\left(\mathbf{p}^{\prime}, r^{\prime}\right)\right|^{2} & =p \cdot p^{\prime}-m^{2} \\
& =\omega(\mathbf{p}) \omega\left(\mathbf{p}^{\prime}\right)-\mathbf{p} \cdot \mathbf{p}^{\prime}-m, \\
u^{*}(\mathbf{p}, r) v(-\mathbf{p}, s) & =0=u^{*}(-\mathbf{p}, r) u(\mathbf{p}, s) .
\end{aligned}
$$

${ }^{1}$ K. O. Friedrichs, Mathematical Aspects of the Quantum Theory of Fields (Interscience, New York, 1953).

${ }^{2}$ L. van Hove, Phys. 18, 145 (1952).

${ }^{3}$ L. Gårding and A. S. Wightman, Proc. Natl. Acad. Aci. (U.S.) 40, 617 (1954).

${ }^{4}$ A. S. Wightman and S. S. Schweber, Phys. Rev. 98, 812 (1955). 
${ }^{5}$ V. Golodes, Usp. Mat. Nauk 2, 122 (1965); 6, 126 (1965) (in Russian).

${ }^{B}$ R. F. Streater and A. S. Wightman, PCT, Spin and Statistics and All That (Benjamin, New York, 1964).

${ }^{7}$ A. S. Wightman, Cargese lectures, 1964.

J. Glimm and A. Jaffe, "A $\lambda \varphi^{\wedge}$ Quantum Field Theory without Cutoffs.I, II, III" (unpublished).

M. Guenin and G. Velo, Helv. Phys. Acta 41, 362 (1968).

$10 \mathrm{~B}$. Gidas, thesis, University of Michigan.

11 J. Glimm, Commun. Math. Phys. 10, 1 (1968)
${ }^{12} \mathrm{~K}$ Hepp, Les Systèmes à un nombre infini de degrés de liberté (C.N.R.S., Paris, 1969)

${ }^{13}$ J. Fabrey, "Exponential Representations of the CCR," thesis, M.I.T., 1969.

14 D. Uhlenbrock, Commun. Math. Phys. 4, 64 (1967).

15 H. Ezawa, J. Math. Phys. 6, 380 (1965).

16 J. Klauder and J. McKenna, J. Math. Phys. 6, 68 (1965); J. Klauder, J. McKenna, and E. Woods, ibid. 7, 822 (1966).

${ }_{17}$ F. Berezin, Methods of Second Quantization (Academic, New York, 1966).

\title{
Microscopic Theory of a Multicomponent System of Charged and Neutral Particles. I. General Quantum Statistical Formulation
}

\author{
Franz Mohling \\ University of Colorado, Department of Physics, Boulder, Colorado 80302 \\ AND \\ Charles Ray Smith* \\ University of Wyoming, Department of Physics, Laramie, Wyoming 82070
}

(Received 28 January 1970)

\begin{abstract}
Recent contributions to the Lee-Yang-Mohling theory of single-component quantum fluids have enabled us to develop a new theory of the quantum statistics for a multicomponent nonrelativistic system of charged and neutral particles in thermal equilibrium. With the emphasis as much as possible on the physical content of the theory, this paper presents the new formulation of quantum statistics with explicit rules for calculating the grand potential and particle and photon momentum distributions. The present formalism not only simplifies and corrects an earlier version, but also it has made possible clear and systematic procedures for resolving some divergence difficulties that occur in the many-body theory of fully ionized gases.
\end{abstract}

\section{INTRODUCTION}

Interest in controlled thermonuclear reactions, stellar atmospheres and interiors, and, more generally, plasmas has focused attention on the physics of fully ionized gases. Although a theory of the nonequilibrium partially ionized gas should be avidly pursued, the more modest goal of developing a precursory theory of the nonrelativistic fully ionized gas in thermal equilibrium is justified in view of the horrendous complexity of the problem. Moreover, a study of the equilibrium properties of a system can provide important information about nonequilibrium systemsfor example, about linear response and transport phenomena.

A few years ago, Mohling and Grandy ${ }^{1}$ developed a formalism for calculating thermodynamic properties, momentum distributions, and pair-correlation functions for a nonrelativistic, multicomponent, fully ionized gas in thermal equilibrium, and that theory has been used in several calculations. ${ }^{2}$ It was later realized that two classes of photon self-energy structures [called $(0,2)$ and $(2,0)$ structures] were accidentally omitted in the self-energy analysis in MG, and it was therefore of interest to amend MG so as to include the missing self-energy structures. However, Mohling, RamaRao, and $\mathrm{Shea}^{3}$ have recently developed a simple and appealing new master-graph theory of a real quantum fluid in thermal equilibrium; the formalism in MRS applies to a single-component quantum fluid (degenerate or nondegenerate) with a short-range interaction. Moreover, Tuttle ${ }^{4}$ has demonstrated that a powerful counterterm technique can be included easily in a quantum statistical theory, such as that of MRS, based upon the Ursell expansion. Thus, rather than revise and correct MG per se, we propose, in this paper, to extend MRS to apply to a multicomponent system of charged and neutral particles and concurrently to incorporate the counterterm technique of Tuttle. The results of our development are expressed in terms of diagrammatic expansions for momentum distributions and the grand potential.

It seems characteristic of any many-body theory to be plagued by divergencies and spurious results. For the systems of interest here, the developments in quantum electrodynamics allow us to take cognizance of some prospective troublesome features of the theory. Thus, from the beginning, we address ourselves to the tasks of renormalizing bare masses of charged particles, of dealing with the infrared problem, and of summing the so-called Coulomb ring diagrams. 